\title{
TAM/c-Met Inhibitor RXDX-106
}

National Cancer Institute

\section{Source}

National Cancer Institute. TAM/C-Met Inhibitor RXDX-106. NCI Thesaurus. Code C150585.

An orally available and selective inhibitor of the receptor tyrosine kinase (RTK) activity of both hepatocyte growth factor receptor (c-Met; HGFR) and receptors in the TYRO3, AXL, and MER (TAM) family, with potential immunomodulating and antineoplastic activities. Upon oral administration of TAM/c-Met inhibitor RXDX-106, this agent selectively targets and binds to TYRO3, AXL, MER and c-Met, and prevents their RT K activity. This blocks TYRO3/AXL/MER/c-Met-mediated signal transduction pathways, and inhibits the proliferation and migration of TYRO3-, AXL-, MER- and c-Met-overexpressing tumor cells. Inhibition of the TAM family in the tumor microenvironment (TME) activates the immune system in the TME, reverses TAM mediated immunosuppression and enhances the anti-tumor immune response, which lead to immune-mediated tumor cell killing. TYRO3, AXL and MER, members of the TAM family of RT Ks, are overexpressed in many tumor cell types. TAMs play key roles in tumor cell proliferation, survival, invasion, angiogenesis and metastasis, and their expression is associated with drug resistance and poor prognosis. c-Met, also overexpressed in many tumor cell types, plays a critical role in tumor formation, proliferation, invasion and metastasis, and contributes to tumor resistance. In the TME, TAM expression on immune cells contributes to tumor cell evasion of immune surveillance and to the negative regulation of immune responses. 